\title{
COPE is differentially expressed in the brain metastases of patients with metastatic breast cancer.
}

Shahan Mamoor, $\mathrm{MS}^{1}$

1shahanmamoor@gmail.com

East Islip, NY USA

Metastasis to the brain is a clinical problem in patients with breast cancer ${ }^{1-3}$. We mined published microarray data 4,5 to compare primary and metastatic tumor transcriptomes to discover genes associated with brain metastasis in patients with metastatic breast cancer. We found that the epsilon subunit of the coatomer protein complex, COPE, was among the genes whose expression was most different in the brain metastases of patients with metastatic breast cancer as compared to normal breast tissues. COPE mRNA was present at increased quantities in brain metastatic tissues as compared to primary tumors of the breast. Up-regulation of COPE expression may contribute to metastasis of tumor cells from the breast to the brain in humans with metastatic breast cancer.

Keywords: breast cancer, metastasis, brain metastases, central nervous system metastases, COPE, systems biology of breast cancer, targeted therapeutics in breast cancer. 
One report described a 34\% incidence of central nervous system metastases in patients treated with trastuzumab for breast cancer ${ }^{2}$. More recently, the NEfERT-T clinical trial ${ }^{6}$ which compared administration of either neratinib or trastuzumab in conjunction with paclitaxel demonstrated that in a randomized, controlled setting, in breast cancer patients treated with neratinib, not only was the incidence of central nervous system recurrence significantly lower, the time to central nervous system metastasis was significantly delayed as compared to patients administered trastuzumab. The alarmingly high rate of central nervous system metastasis described, as well as data, both anecdotal ${ }^{2}$ and from a randomized, controlled setting ${ }^{6}$ illustrating that treatment with trastuzumab may be associated with these events demands an enhanced understanding of the transcriptional makeup of brain metastatic tissues to support identification of therapeutic targets, whether they are treatment related or not. We performed a global comparative analysis of the benign breast, primary and metastatic tumors in patients with brain metastatic breast cancer ${ }^{4,5}$. We discovered significant differential and increased expression of COPE in brain metastatic tissues of patients with metastatic breast cancer.

\section{Methods}

We used datasets GSE108934 and GSE526045 for this global differential gene expression analysis of brain metastatic breast cancer in conjunction with GEO2R. GSE10893 was generated using Agilent-011521 Human 1A Microarray G4110A technology with $n=11$ primary breast tumors and $n=4$ brain metastases from patients with breast cancer; analysis performed using platform GPL885. GSE52604 was generated using Whole Human Genome Microarray 4x44K G4112F technology with $\mathrm{n}=10$ normal breast tissues and $\mathrm{n}=35$ brain metastases from patients with breast cancer, analysis performed using platform GPL6480. The Benjamini and Hochberg method of $p$-value adjustment was used for ranking of differential expression but raw $p$-values were used to assess statistical significance of global differential expression. Log-transformation of data was auto-detected, and the NCBI generated category of platform annotation was used. A statistical test was performed to evaluate whether COPE gene expression was significantly between primary tumors of the breast and brain metastases in humans with breast cancer using a two-tailed, unpaired t-test with Welch's correction. We used PRISM for all statistical analyses of differential gene expression in human breast cancer (Version 8.4.0)(455).

\section{Results}

We performed global comparative transcriptome analysis of metastatic tumor tissues of patients with metastatic breast cancer using published microarray data ${ }^{4,5}$ to describe the transcriptional landscape of brain metastasis in human breast cancer in an unbiased fashion and for the discovery of novel therapeutic targets.

\section{COPE is differentially expressed in the brain metastases of patients with brain metastatic breast} cancer.

Through analysis of published microarray data ${ }^{4}$, we identified the epsilon subunit of the coatomer protein complex, encoded by COPE, as a differentially expressed gene in the breast metastatic tissues of humans with breast cancer. When sorting each of the genes expressed in brain metastases based on significance of difference as compared to primary tumors of the breast in patients with breast cancer, COPE 68 out of 17418 total transcripts (Table 1), equating to $99.6 \%$ differential expression. Differential expression of COPE in the brain metastases of patients with metastatic breast cancer was statistically significant (Table $1 ; p=1.26 \mathrm{E}-04$ ).

Identification of differentially expressed genes across datasets in cancer can be difficult and more challenging than when doing so in benign organ tissue and sorted cell populations due to higher levels of heterogeneity in tumors, and differing methods of tumor sampling between laboratories. Thus, we asked 
how gene expression was most significantly different between brain metastases from patients with breast cancer and normal breast tissue as opposed to primary tumors of the breast, by comparing global gene expression profiles of 10 normal breast tissues to 35 brain metastases 5 . Using this strategy, we validated COPE as a differentially expressed gene in the brain metastases of patients with brain metastatic breast cancer ${ }^{4}$ (Table 2). When sorting each of the genes expressed in brain metastases based on significance of difference in expression between brain metastases and normal breast tissues, COPE ranked 379 out of 41093 total transcripts (Table 2), equating to $99.1 \%$ differential expression. Differential expression of COPE in brain metastases from patients with brain metastatic breast cancer in this dataset was statistically significant (Table $2 ; p=2.76 \mathrm{E}-09$ ).

Thus, differential expression of COPE was conserved both when compared to normal tissues of the breast and to primary tumors of the breast in patients with metastatic breast cancer, in two independent microarray datasets 4,5

COPE is expressed at higher levels in the brain metastases of patients with brain metastatic breast cancer.

We obtained exact mRNA expression levels for COPE, in primary tumors of the breast and in brain metastasis of patients with brain metastatic breast cancer to determine direction and statistical significance of change in COPE expression in brain metastatic tissues. COPE was expressed at higher levels in the brain metastases of patients with breast cancer as compared to primary tumors of the breast, but this difference was not deemed statistically significant (Figure $1 ; p=0.0034$ ).

Thus, by mining published microarray data 4,5 in an unbiased and systematic fashion, we identified COPE as among the genes whose expression was most different, transcriptome-wide, in the brain metastases of patients with breast cancer both when compared to the normal breast and to primary tumors of the breast; COPE expression, as compared to primary tumors of the breast, was higher in metastasis to the brain.

\section{Discussion}

We provided evidence here that the epsilon subunit of the coatomer protein complex encoded by COPE is among the genes whose expression is most different in the brain metastases of patients with brain metastatic breast cancer. Evaluation of the phenotypic consequences of genetic depletion of COPE in mouse models of metastatic breast cancer on metastasis to the central nervous system is merited. 


\section{References}

1. Lin, N.U., Amiri-Kordestani, L., Palmieri, D., Liewehr, D.J. and Steeg, P.S., 2013. CNS metastases in breast cancer: old challenge, new frontiers.

2. Bendell, J.C., Domchek, S.M., Burstein, H.J., Harris, L., Younger, J., Kuter, I., Bunnell, C., Rue, M., Gelman, R. and Winer, E., 2003. Central nervous system metastases in women who receive trastuzumab-based therapy for metastatic breast carcinoma. Cancer, 97(12), pp.2972-2977.

3. Tsukada, Y., Fouad, A., Pickren, J.W. and Lane, W.W., 1983. Central nervous system metastasis from breast carcinoma autopsy study. Cancer, 52(12), pp.2349-2354.

4. Weigman, V.J., Chao, H.H., Shabalin, A.A., He, X., Parker, J.S., Nordgard, S.H., Grushko, T., Huo, D., Nwachukwu, C., Nobel, A. and Kristensen, V.N., 2012. Basal-like Breast cancer DNA copy number losses identify genes involved in genomic instability, response to therapy, and patient survival. Breast cancer research and treatment, 133(3), pp.865-880.

5. Salhia, B., Kiefer, J., Ross, J.T., Metapally, R., Martinez, R.A., Johnson, K.N., DiPerna, D.M., Paquette, K.M., Jung, S., Nasser, S. and Wallstrom, G., 2014. Integrated genomic and epigenomic analysis of breast cancer brain metastasis. PloS one, $9(1)$, p.e85448.

6. Awada, A., Colomer, R., Inoue, K., Bondarenko, I., Badwe, R.A., Demetriou, G., Lee, S.C., Mehta, A.O., Kim, S.B., Bachelot, T. and Goswami, C., 2016. Neratinib plus paclitaxel vs trastuzumab plus paclitaxel in previously untreated metastatic ERBB2-positive breast cancer: the NEfERT-T randomized clinical trial. JAMA oncology, 2(12), pp.1557-1564. 
Table 1: COPE is differentially expressed in brain metastases of patients with metastatic breast cancer when compared to primary tumors of the breast.

Rank of differential expression, probe ID, $p$-value with respect to differential expression, $\mathrm{t}$, a moderated tstatistic, B, the log-odds of differential expression between the two groups compared, gene and gene name are listed in this chart.

PAGE 5 


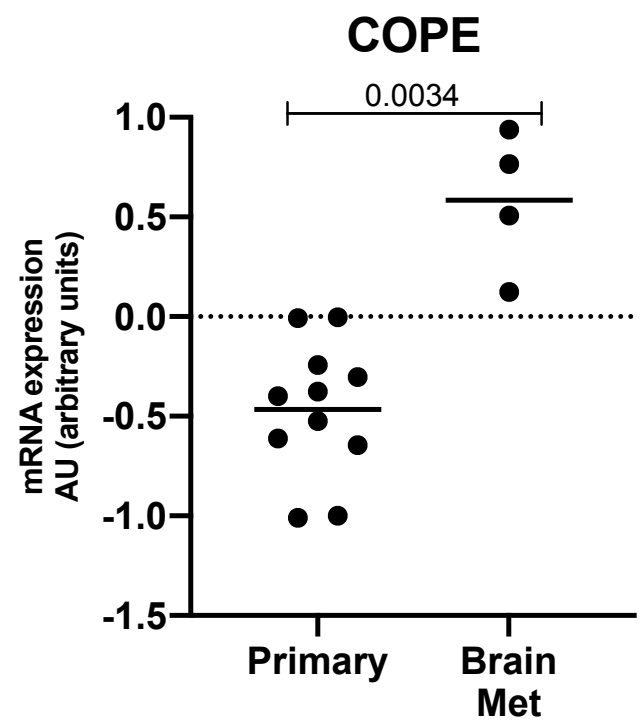

Figure 1: COPE is expressed at higher levels in the brain metastases of patients with brain metastatic breast cancer as compared to primary tumors of the breast.

The mRNA expression level of COPE in primary tumors of the breast (left) and in the brain metastases of brain metastatic breast cancer patients (right) is graphically represented here with mean COPE levels marked and the result of a statistical test evaluating difference in mRNA expression level between brain metastases and primary tumors of the breast, a $p$-value, listed above. 